\title{
DIGITAL HOLOGRAPHIC LOGIC
}

\author{
K. Preston, Jr. \\ The Perkin-Elmer Corporation, Norwalk, Connecticut 06856

\section{$N 73-18896$}

INTRODUCTION

Optical computers ordinarily operate in an analog mode where signals introduced into the computer take on continuous values. The computing rate achievable in such computers is given by

$$
\mathrm{R}_{\mathrm{c}}=\frac{\epsilon \eta \mathrm{P}}{\mathrm{h} v(\mathrm{~S} / \mathrm{N})^{2}}
$$

where $\epsilon$ is the optical transfer efficiency, $\eta$ is the quantum efficiency of the output detector, $\mathrm{P}$ is the optical input power, $\mathrm{h}$ is Planck's constant, $v$ is the optical frequency, and $(\mathrm{S} / \mathrm{N})$ is the desired $\mathrm{rms}$ output signal to noise ratio.

For typical values such as $\epsilon=0.5, \eta=0.1, \mathrm{P}=1.0$ watt, $\nu=10^{15} \mathrm{~Hz}, \mathrm{~S} / \mathrm{N}=10, \mathrm{R}_{\mathrm{c}}$ is of the order of $10^{14}$ multipications per second. Accuracy, however, is low. In ordinary optical systems the accuracy level is 5 bits, but with extreme care in both optical design and lens element fabrication, 7 to 8 bit accitracy may be achieved (1).

Ordinarily the dynamic range in the optical analog computer is $40 \mathrm{db}$ (13bits). Again, with extreme care in design, fabrication and assembly $60 \mathrm{db}$ (20-bits) may be reached. In comparison with the electronic digital computer, where in many cases 128 bits accuracy is available for double precision arithmetic, the optical analog computer is far from competitive.

However, it is possible for optical computers to achieve high accuracy by operating in a digital mode using signal levels which are restricted to the binary values $(0$ and 1$)$. The purpose of this paper is to describe experiments conducted at Perkin-Elmer demonstrating the feasibility of an optical digital computer. The program of this computer is entered holographically. This would be done under control of an electronic digital control unit (see Figure 1). The digital control unit would store the program and would also act as a storage medium for binary number arrays. These arrays would form the input to the optical computer and similar arrays would be retrieved from the optical computer when calculations were completed.

In order to satisfactorily combire the relatively slow electronic digital control unit with the optical computer, it is necessary that the optical computer iterate through many stages of a calculation before either requesting more data 


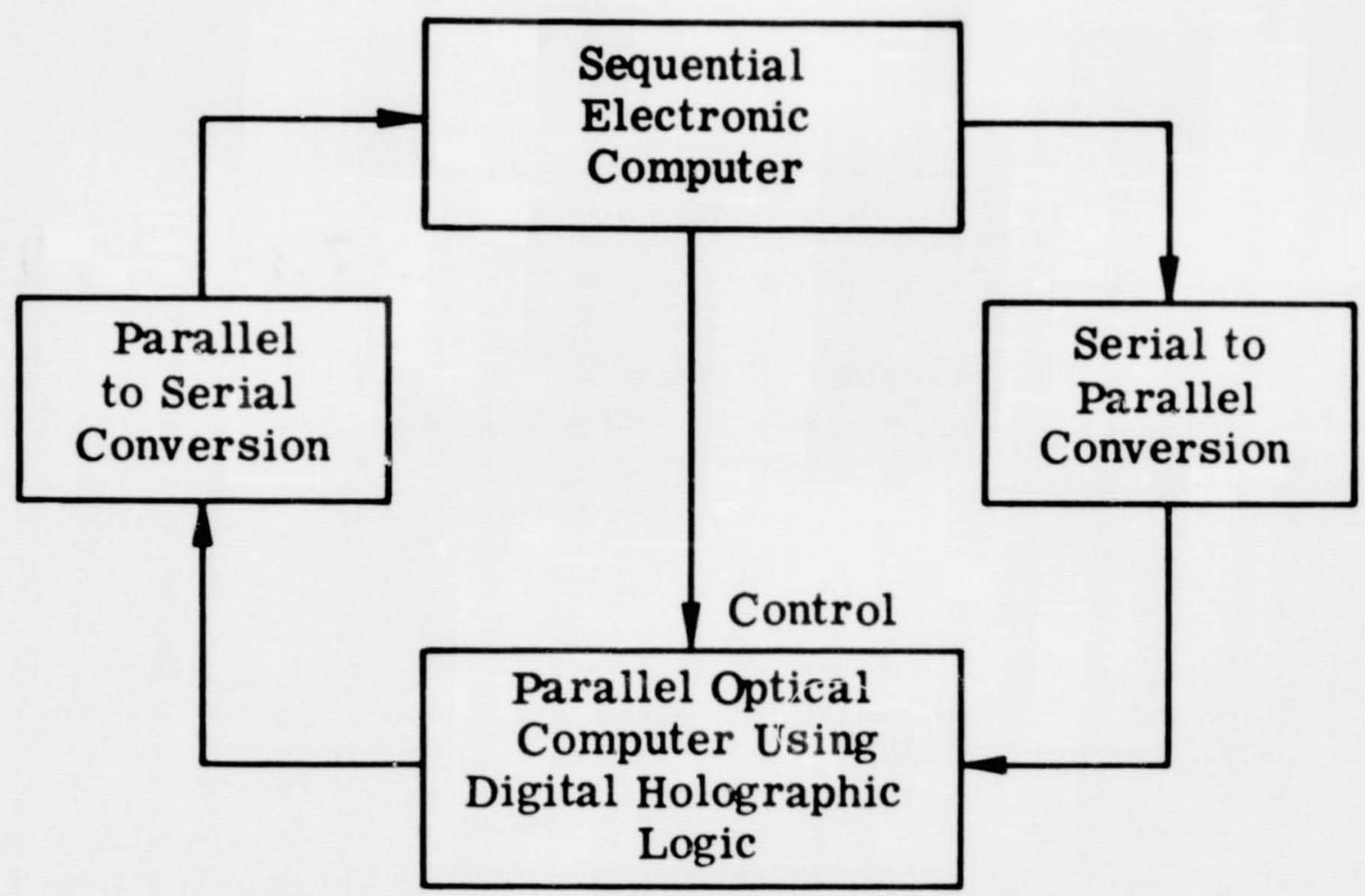

Figure 1. Block schematic showing connections between the electronic control computer and the parallel optical computer for use in performing digital logic holographically.

at its input or transferring results from its output. The optical computer would thus be built from a concatenation of blocks of the type shown in Figure 2. The block shown in this figure consists of a combination of optical-to-optical conversion devices (OTTO's) interspersed with computing elements consisting of lens pairs and holograms.

This paper discusses the characteristics of the block shown in Figure 2. First the holographic operation is reviewed from the Fourier transform viewpoint. The paper then describes the formation of holograms for use in performing digital logic, illustrates the operation of the computer with an experiment in which the binary identity function is calculated, then discusses devices for achieving real-time performance, and describes an application in pattern recognition using neighborhood logic,

\section{HOLOGRAPHY}

Figure 3 shows a schematic representation of the first stage of the block shown in Figure 2. This block makes use of the lens in taking the Fourier 


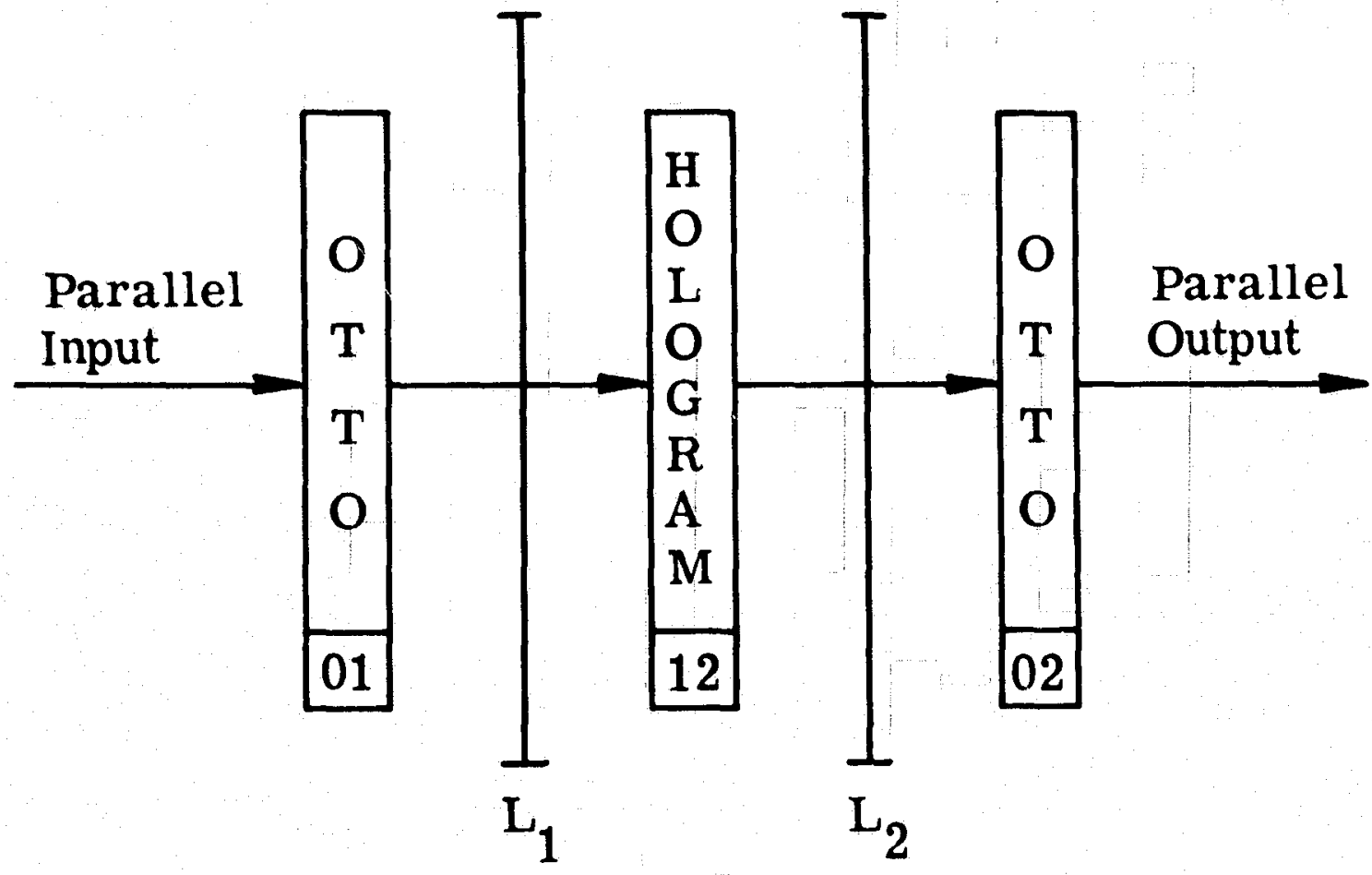

Figure 2. Schematic arrangement of the basic building block of the holographic logic computer. Two optical to optical transducers, one hologram, and two Fourier transform lenses are required.

transform. The $x_{1}$ plane is the input plane, $L_{12}$ is the transform lens, and the $x_{2}$ plane is the Fourier transform plane. These planes are separated by two focal lengths. The lens action of the transform lens is given in the below equation (2).

$$
E\left(x_{2}\right)=\int E\left(x_{1}\right) e^{-j \omega_{x} x_{1}} d x_{1}
$$

where $E\left(x_{1}\right)$ is the electric field distribution in the input plane, $E\left(x_{2}\right)$ is the electric field distribution in the output plane and $\omega_{x}=2 \pi x_{2} / \lambda F$ where $\lambda$ is the optical wavelength and $F$ is the focal length of $L_{12}$.

Assume that the $x_{1}$ plane contains a spatial light modulating device whose light modulating elements are discrete and regular in position. One such element is located at $x_{i a}$. Light emanating from this point may be considered to be a positional delta function which is transformed into a plane wave in the $x_{2}$ plane as follows.

$$
E\left(x_{2}\right)=\int \delta\left(x_{1}-x_{a}\right) e^{-j \omega x_{1}} d x_{1}=e^{-j \omega} x_{a}
$$




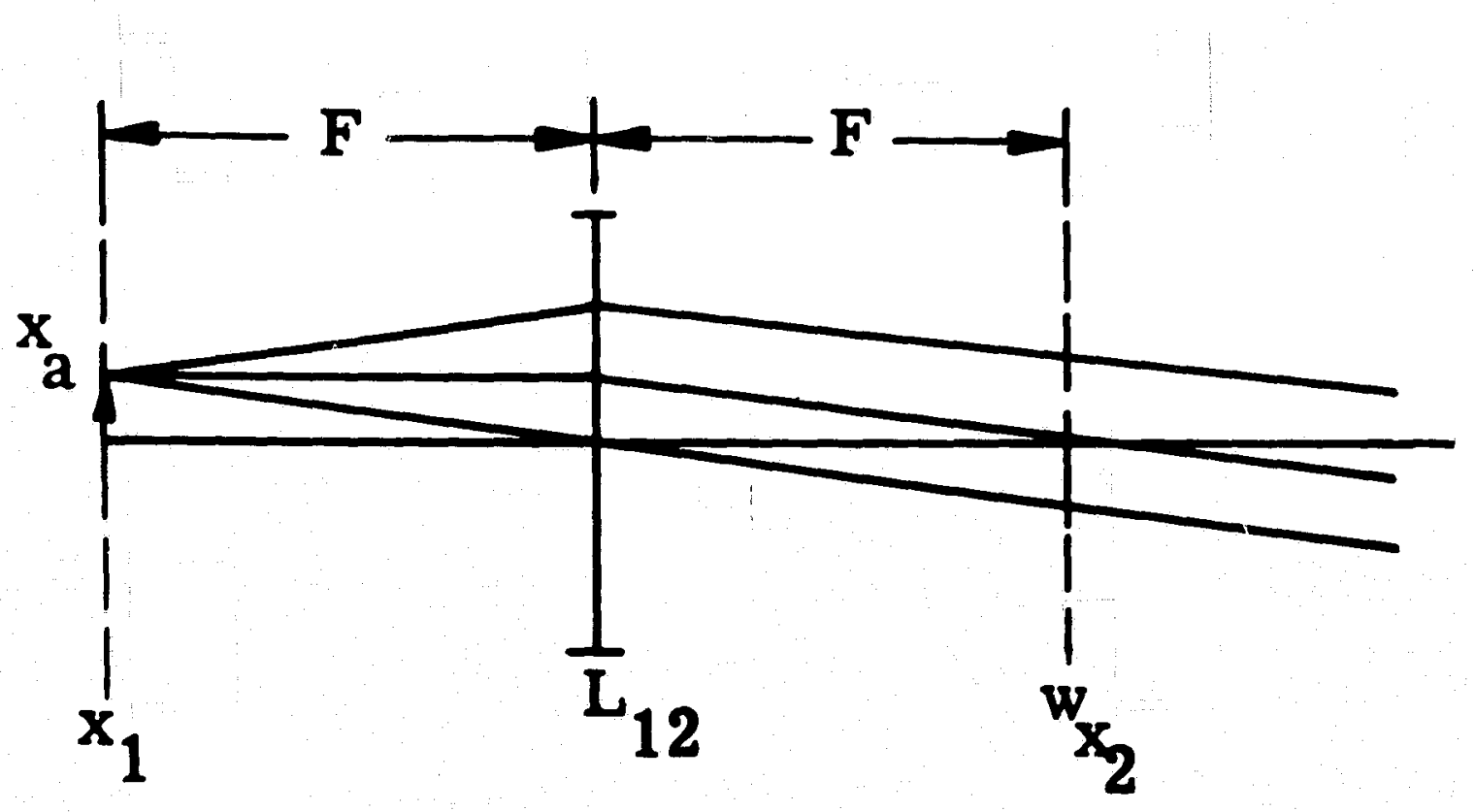

$$
\begin{aligned}
E\left(w_{x_{2}}\right) & =\int E\left(x_{1}\right) e^{-j w x_{2} x_{1}} d x_{1} \\
w_{x_{2}} & =2 \pi x_{2} / \lambda F \\
\delta\left(x_{1}-x_{a}\right) & \longrightarrow e^{-j w_{x_{2}} x_{a}}
\end{aligned}
$$

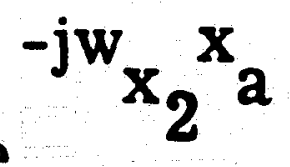

Figure 3. Analytical relationship between a discrete input at $x_{a}$ in the input plane and the associated plane wave in the Fourier plane created by the transform lens $L_{12}$.

When the second lens of the block is introduced, the block is complete and the configuration is as shown in Figure 4. The lens $L_{23}$ takes a Fourier transform with the result appearing in the $x_{3}$ plane. The reader should note that, since lenses in optical computers take successive Fourier transforms, the 


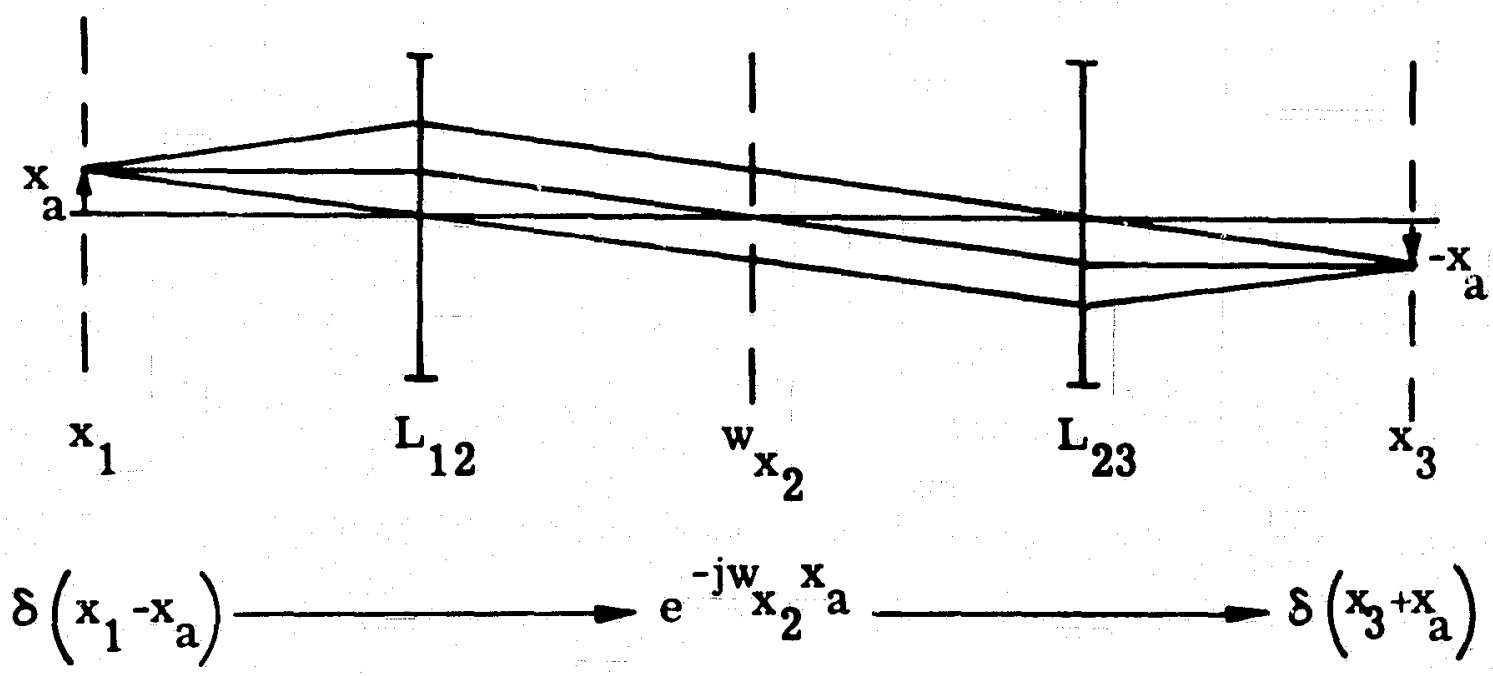

Figure 4. Analytical relationship between the discrete input at $x_{a}$ in the input plane and its image at $-x_{a}$ in the output plane when two successive Fourier transforms are taken using lenses $L_{12}$ and $L_{23}$.

inverse of the input function appears at the output, namely, a delta function not at $x_{a}$ but at $-x_{a}$. This is expressed in the following equations

$$
\begin{aligned}
E\left(x_{3}\right) & =\int E\left(x_{2}\right) e^{-j \omega_{x_{2}}} d x_{2} \\
& =\int e^{-j \omega_{x_{3}}^{\prime}\left(x_{3}+x_{a}\right)} d x_{2} \\
& =\delta\left(x_{3}+x_{a}\right)
\end{aligned}
$$

where $\omega_{x}=2 \pi x_{3} / \lambda F$ and $\omega_{x}^{\prime}=2 \pi x_{2} / \lambda F$.

In order to form a hologram and illustrate its operation upon an input delta function at $x_{a}$ consider the configuration diagrammed in Figure 5. Two discrete inputs are provided: one at $x_{a}$; one at $x_{r}$. Lens $L_{12}$ carries out the Fourier transform of these two delta functions to produce the electric field distribution

$$
E\left(x_{2}\right)=e^{-j \omega_{x} x_{a}}+e^{-j \omega_{x} x_{r}}
$$




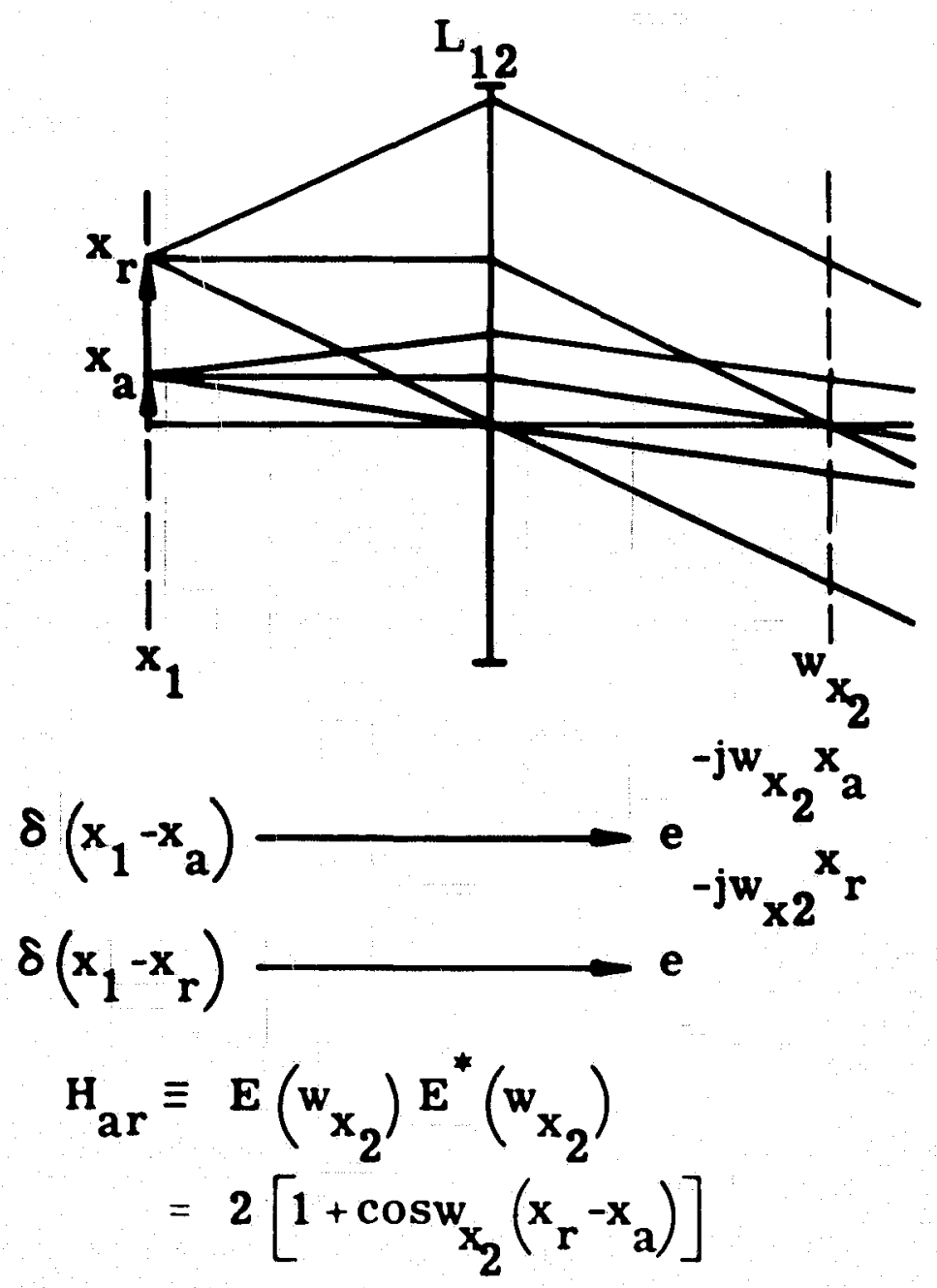

Figure 5. Equations describing the generation of a hologram in the Fourier plane using a discrete input at $x_{a}$ and $a$ reference at $x_{r}$ in the input plane.

An energy detector placed in the $x_{2}$ plane records the hologram $H_{\text {ar }}$ given as follows

$$
H_{a r}=E\left(x_{2}\right) E^{*}\left(x_{2}\right)=2\left[1+\cos \omega_{x}\left(x_{r}-x_{a}\right)\right]
$$

Note that this hologram consists of a bias term plus a cosinusoidal term whose spatial frequency is proportional to the quantity $\left(x_{r}-x_{n}\right)$.

When this hologram is placed in the optical computer and a discrete input occurs at $x_{a}$, the result is as shown in Figure 6 . Once again the lens $L_{12}$ generates a plane wave in the $x_{2}$ plane in which the hologram $H_{\text {ar }}$ is located. The 


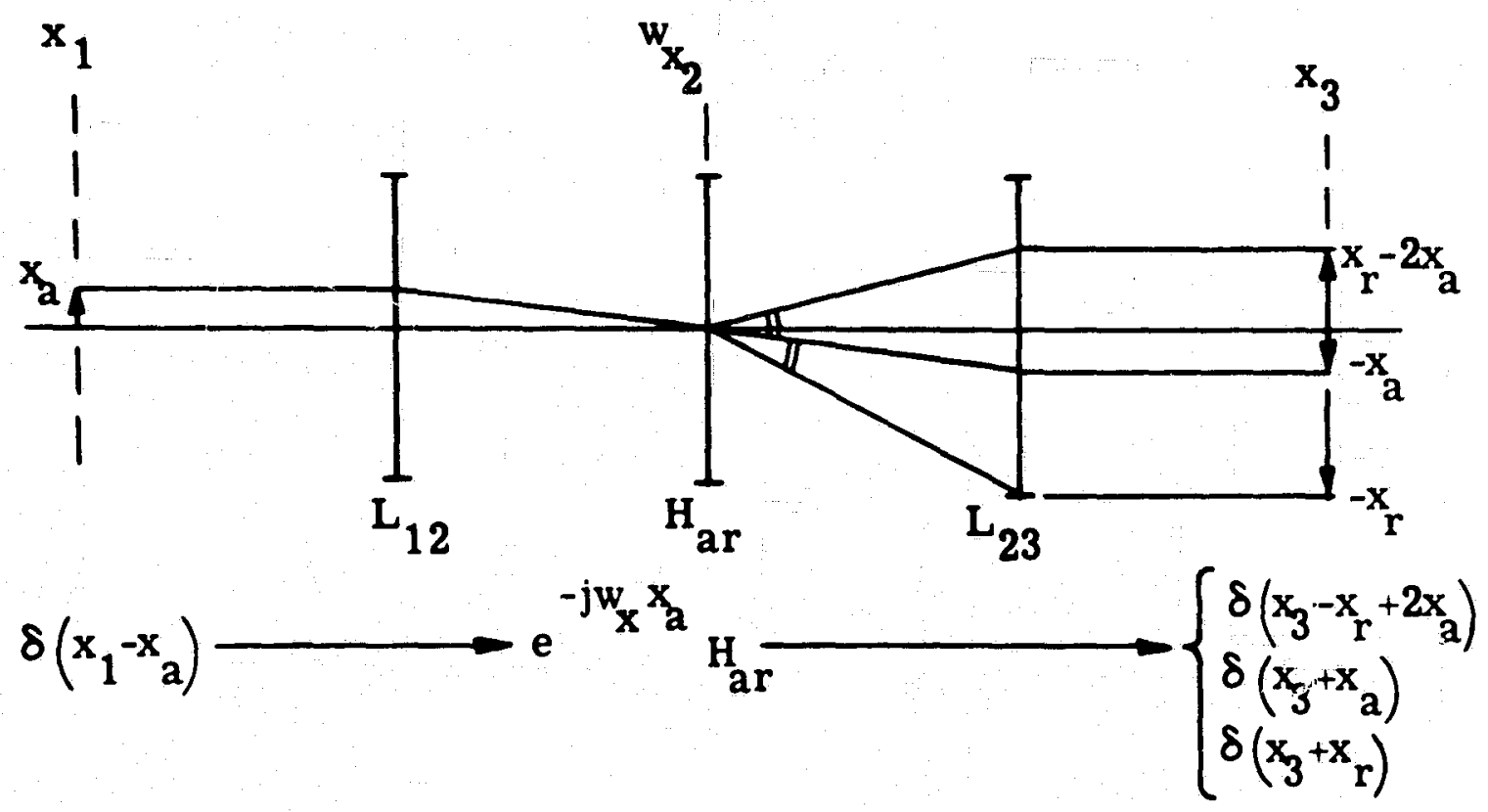

Figure 6. Schematic showing the results when a discrete input at $x_{a}$ is used in conjunction with a hologram $\mathrm{H}_{\mathrm{ar}}$ in the Fourier plane to produce three discrete outputs in the output plane.

plane wave interacts with $\mathrm{H}_{a \mathrm{r}}$ in such a way that three plane waves are created which are transformed by $\mathrm{L}_{23}$ into three delta functions according to the below equation

$$
\begin{aligned}
E\left(x_{3}\right) & =\int\left[2+e^{j \omega_{x}\left(x_{r}-x_{a}\right)}+e^{-j \omega_{x}\left(x_{r}-x_{a}\right)} e^{-j \omega_{x} x_{a}} e^{-j \omega_{x} x_{2}} d x_{2}\right] \\
& =\int\left[2 e^{-j \omega_{x}^{\prime}\left(x_{3}+x_{a}\right)}+e^{-j \omega_{x}^{\prime}\left(x_{3}+x_{r}\right)}+e^{-j \omega_{x}^{\prime}\left(x_{3}-x_{r}+2 x_{a}\right)} d x_{2}\right] \\
& =2 \delta\left(x_{3}+x_{a}\right)+\delta\left(x_{3}+x_{r}\right)+\delta\left(x_{3}-x_{r}+2 x_{a}\right)
\end{aligned}
$$

The reader familiar with matched filter theory will recognize that the delta function at $-x_{r}$ is the peak of the correlation function generated by $H_{a r}$ acting as a matched fiiter in the frequency domain (3).

Another way of diagramming the action of $\mathrm{H}_{\text {ar }}$ is shown in the left hand portion of Figure 7. This simplified diagram indicates only the input plane (horizontal line at top) and the output plane (horizontal line at bottom). The action of the entire lens system in combination with $\mathrm{H}_{\text {ar }}$ is shown merely by the dowriward 


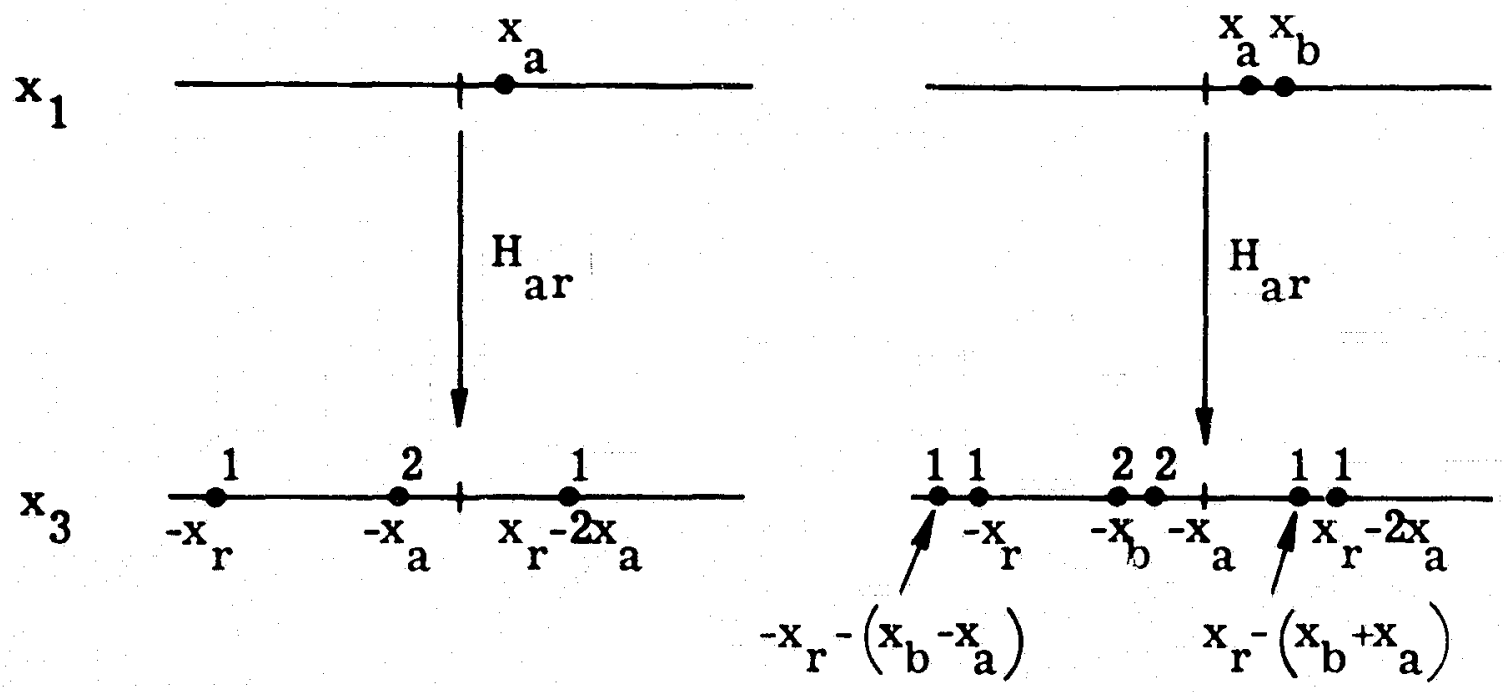

Figure 7. Abbreviated schematics showing (left hand drawing) the result given in Figure 6 and (right hand drawing) result when using two discrete inputs in the input plane at the points $x_{a}$ and $x_{b}$.

directed arrow. On the line representing the output plane the numerics indicate the electric field strength of three delta functions. Note that the electric field strength at $-\mathrm{x}_{\mathrm{a}}$ is twice that of the other two delta functions.

\section{HOLOGRAPHIC LOGIC}

In performing holographic digital logic it is important to understand how the hologram interacts with light emanating from a multiplicity of discrete inputs in the $x_{1}$ plane. The right hand portion of Figure 7 shows what occurs when a second input is added at $x_{b}$. New delta functions appear in the output plane separated by the distance $\left(x_{b}-x_{n}\right)$ from the other delta functions. They are of identical electrical field strength and of equal phase if we assume that the input at $x_{b}$ has strength and phase equal to that at $x_{a}$.

In order to perform digital logic holographically it is necessary to combine at one point in the output plane energy emanating from two discrete inputs in the $x_{1}$ plane. This may be done by using a somewhat more complex hologram constructed in the same manner as shown in Figure 5 but with discrete inputs at both $x_{a}$ and at $x_{b}$ simultaneously. The electric field strength in the $x_{2}$ plane is given by the equation

$$
E\left(x_{2}\right)=e^{-j \omega_{x} x_{a}}+e^{-j \omega x_{0}}+e^{-j \omega_{x} x_{r}}
$$




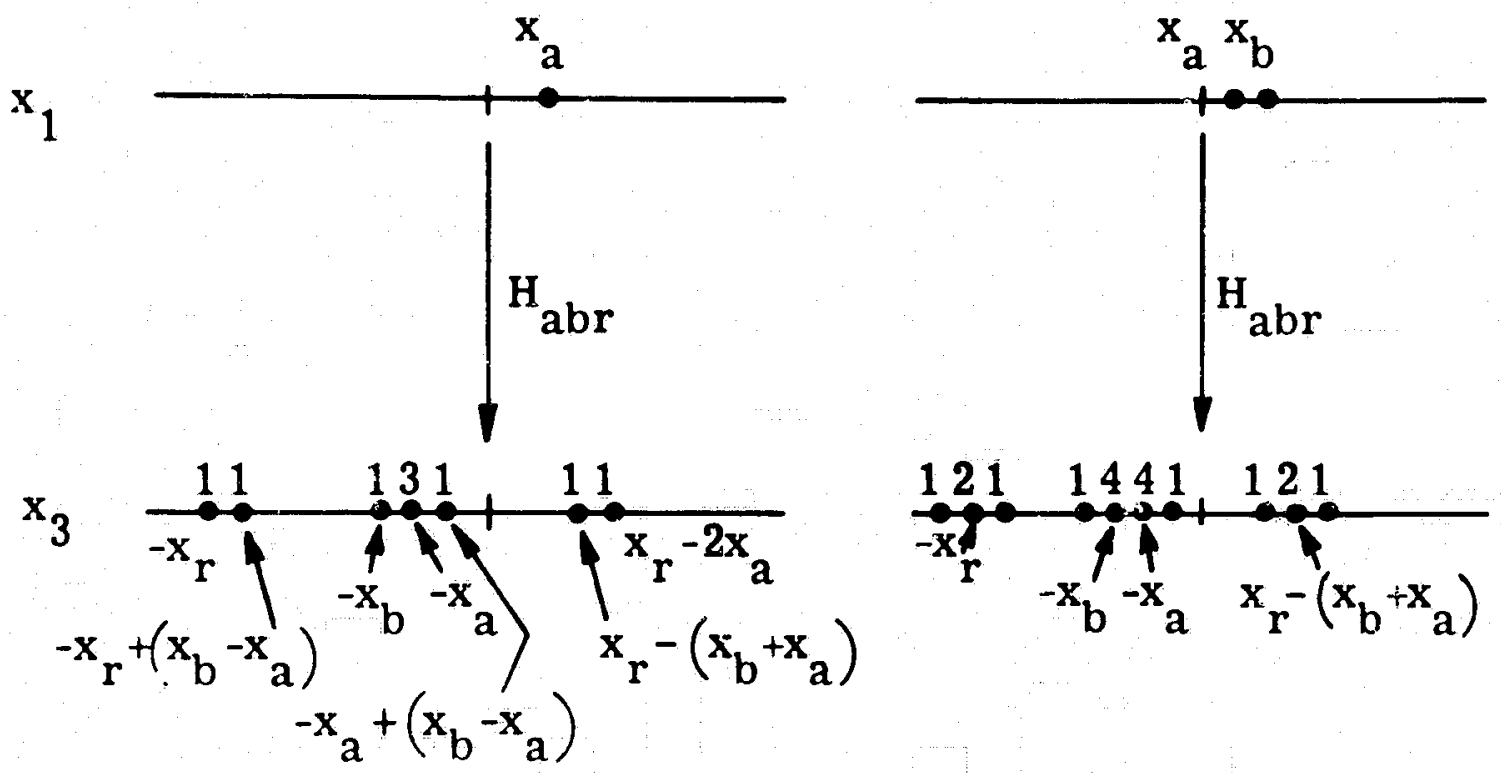

Figure 8. Abbreviated schematics showing the results of using the hologram $H$ in the Fourier plane with a single discrete input at $x_{a}$ (left hand drawing) with two discrete inputs at the points $x_{a}$ and $x_{b}$ (right hand drawing).

and the hologram $\mathrm{H}_{\mathrm{abr}}$ resulting from exposing an energy detector to this field is given by

$$
\begin{aligned}
H_{a b r} & =E\left(x_{2}\right) E^{*}\left(x_{2}\right) \\
& =3+2\left[\cos \omega_{x}\left(x_{r}-x_{a}\right)+\cos \omega_{x}\left(x_{r}-x_{b}\right)+\cos \omega_{x}\left(x_{b}-x_{a}\right)\right]
\end{aligned}
$$

As can be seen there are three cosinusoidal terms. If we a.ssume, as is usually the case, that the reference input at $x_{r}$ is considerably removed from the optical axis, then two of the cosinusoidal terms represent high spatial frequencies while the other has a relatively low spatial frequency related to the difference in position between $x_{b}$ and $x_{a}$ ).

When hologram $\mathrm{H}_{\mathrm{abr}}$ is introduced in the $\mathrm{x}_{2}$ plane and an input at $\mathrm{x}_{\mathrm{a}}$ is present in the $x_{1}$ plane, the resultant output electric field distribution is shown in the left hand side of Figure 8 . The reader should compare this result with that shown in the left hand side of Figure 7 which relates to the same input configuration when using hologram $\mathrm{H}_{\mathrm{ar}}$.

When using hologram $\mathrm{H}_{\mathrm{abr}}$ a single iruput in the $\mathrm{x}_{1}$ plane produces a holographic image of the input plane configuration for which $\mathrm{H}_{\mathrm{abr}}$ is the hologram. Thus not only does an output delta function appear at $-x_{r}$ but there is another 


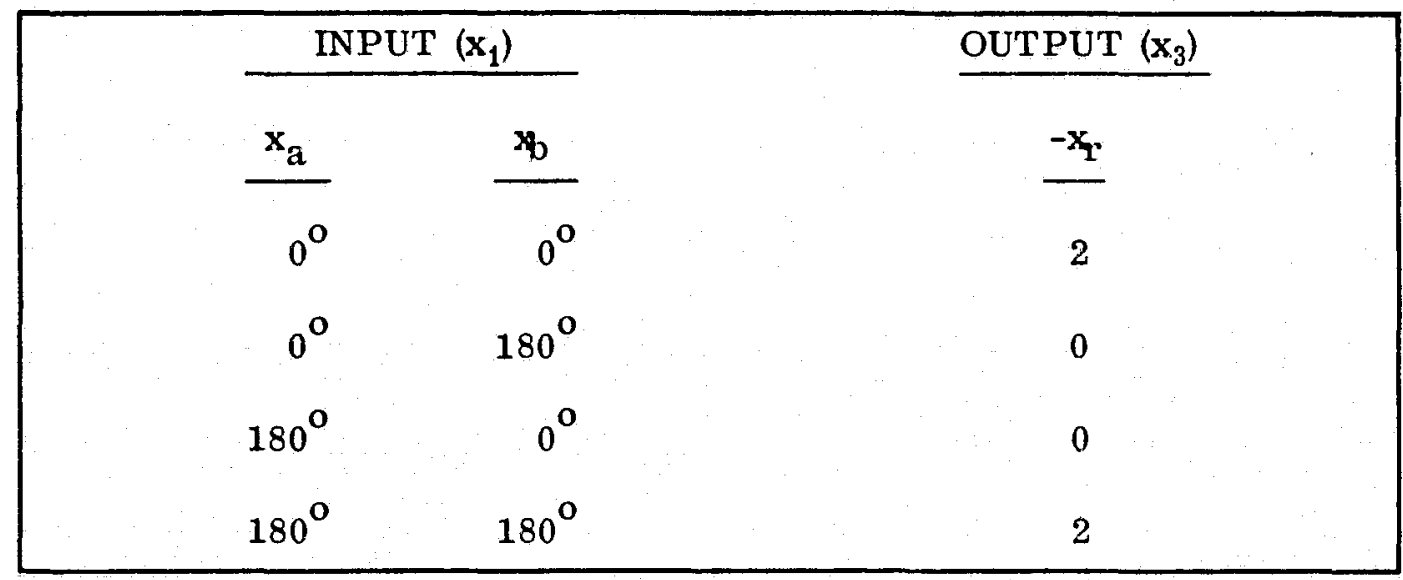

Figure 9. Electric field strength in the output plane at the point $-x_{r}$ produced using each of the four binary combinations of inputs at $x_{a}$ and $x_{b}$.

delta function at a distance $\left(x_{b}-x_{a}\right)$ from it. Holographically speaking this is the "reconstruction" of the input configuration for which $H_{a b r}$ is the hologram (4).

The final and most important result is shown on the right hand side of Figure 8. Here the configuration of inputs in the $x_{1}$ plane is identical to that from which the hologram $\mathrm{H}_{\mathrm{abr}}$ is made. When this is the case, the electric field strength at the point $-x_{r}$ in the output plane is the coherent summation of the electric fields at $x_{a}$ and $x_{b}$ in the input plane. Speaking from the point of view of matched filter theory, the electric field strength in the vicinity of $-x_{r}$ is the correlation function of the input configuration whereas that in the vicinity of the conjugate region at $\left[x_{r}-\left(x_{b}+x_{i 1}\right)\right]$ is the convolution function. Other inputs appear near the optical axis. These have the highest electric field and occur where the input configuration is imaged by the combined action of $\mathrm{L}_{12}$ and $\mathrm{L}_{23}$.

In using holograms stich as $\mathrm{H}_{\mathrm{abr}}$ for performing digital holographic logic only the delta functions near $-x_{r}$ is of importance. Therefore in the discussion which follows only this region of the output plane is treated.

\section{THE BINARY IDENTITY FUNCTION}

In order to perform digital logic using $H_{a b r}$ it is necessary to elect a method for introducing the binary values of the input variables. The most straightforward approach is to use zero phase for the binary 0 and the opposite phase $\left(180^{\circ}\right)$ for the binary 1 . The resultant field strength in the output plane at the point $-x_{r}$ for this selection of the binary variables at $x_{a}$ and $x_{b}$ is tabulated in Figure 9. In all cases it is assumed that the electric field strengths at $x_{i 1}$ and $x_{b}$ are identical. As can be seen, when the phase of the electric field at $x_{a}$ and 
$x_{b}$ are the same, the output field strength at $-x_{r}$ is finite. When the phases are opposite, the output at $-x_{r}$ is zero. Optically speaking, finite and zero outputs correspond to constructive and destructive interference, respectively. Logically speaking, the tabulation shown on Figure 9 may be considered to be a truth table for the identity function.

In order to completely appreciate the appearance of the output plane, refer to Figure 10 which shows in the two left hand columns the states of the input variables and in the three right hand columns the complete correlation function configuration in the vicinity of the point $-x_{r}$. Not only is the identity function (center column) shown but also the light distribution in its immediate vicinity for all four possible pairs of values of the input variables.

\section{EXPERIMENTAL CONFIRMATION}

An experiment was recently carried out in the laboratory where such a computation was performed using a hologram of the type given by $\mathrm{H}_{a b r}$. Specifically this hologram was created using two discrete inputs consisting of $50 \mu \mathrm{m}$ diameter pinholes with a $100 \mu \mathrm{m}$ separation. An input light modulator was then created by vapor deposition which is shown in Figure 11. This light modulator

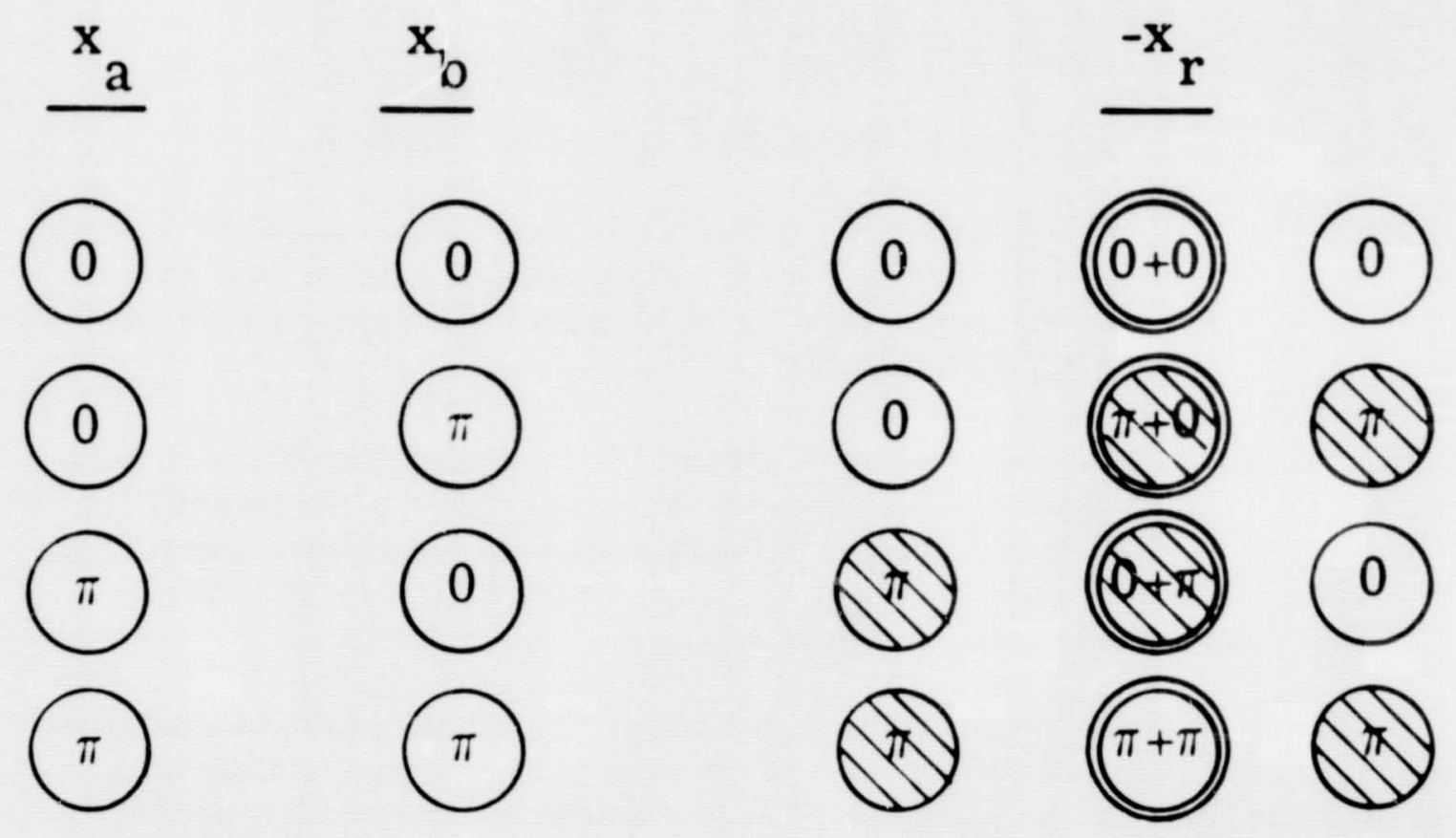

Figure 10. Diagram of the autocorrelation functions produced in the vicinity of the point $-x_{r}$ in the output plane for the four possible binary combinations of inputs at points $x_{a}$ and $x_{b}$. 


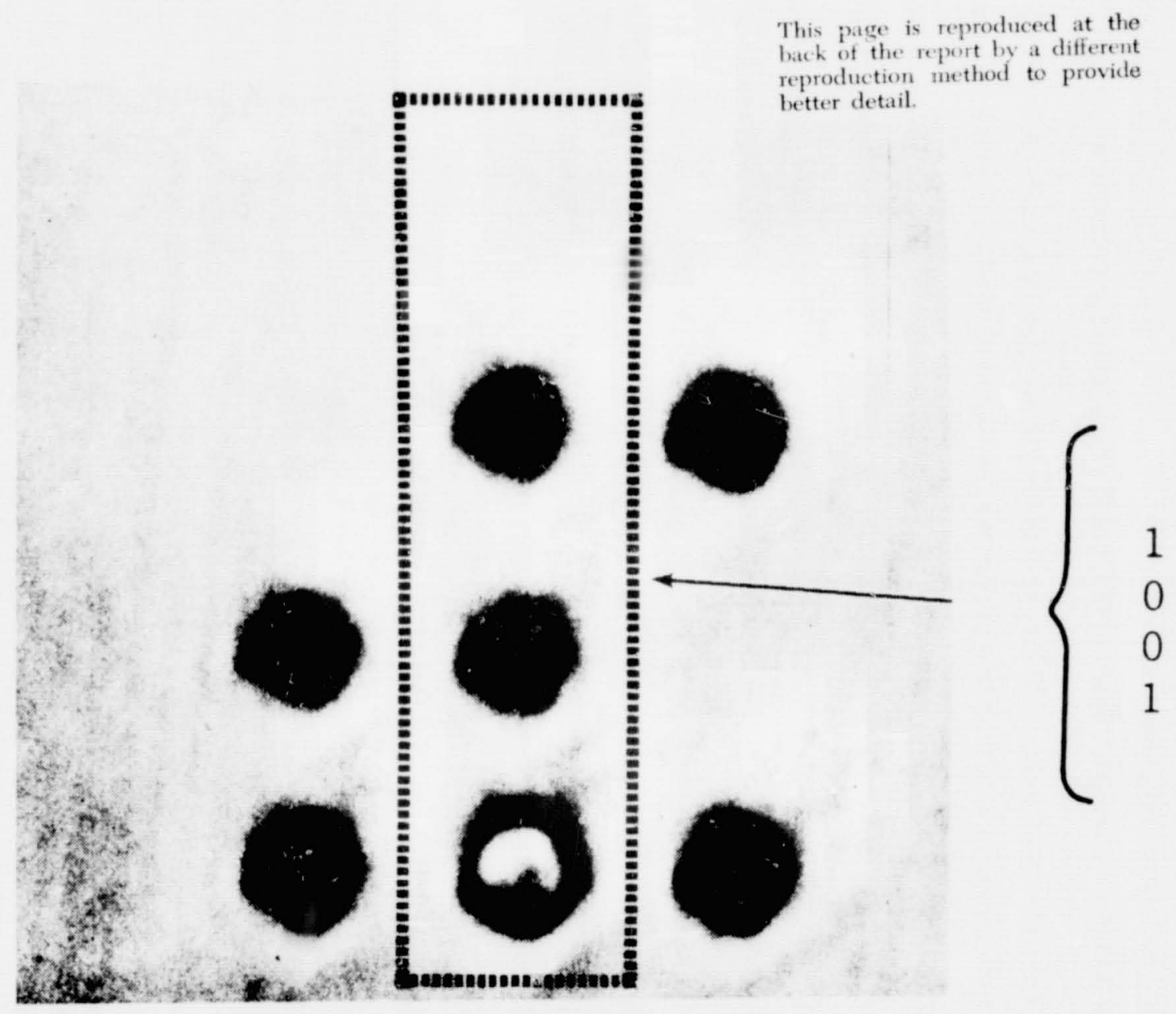

Figure 11. Photomicrograph of a two dimensional spatial light modulator having four phase modulating regions (encircled) whose action is to shift the phase of transmitted light by 180 degrees with respect to light passing through adjacent regions.

had its elements on a grid spacing of $100 \mu \mathrm{m}$. Each element has $50 \mu \mathrm{m}$ in diameter. Four elements were created which shifted the phase of the transmitted light by $180^{\circ}$ with respect to light passing through the background. These light modulating elements were arranged so that they would correspond to the input variable combinations given in Figure 10.

Figure 12 shows the resultant light intensity distribution in the output plane when the light modulator was placed in the input plane. There is a background of in-phase illumination. Regions $50 \mu \mathrm{m}$ in diameter showing both constructive and destructive interference may also be observed. In the central column the expected binary identification function is displayed. The "bright" output expected for the fourth value of the identity function is surrounded by a dark halo due to 


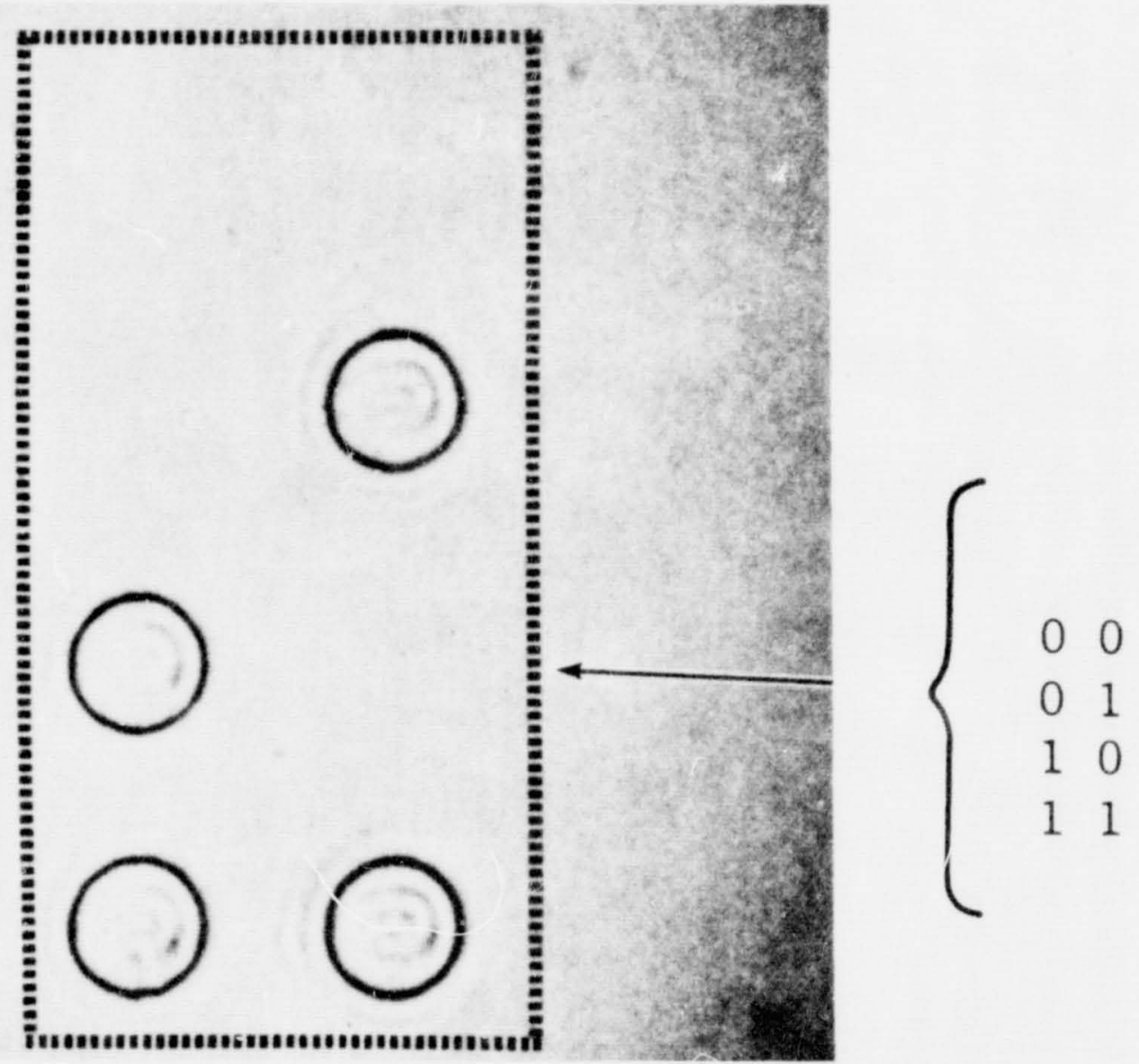

Figure 12. Photomicrograph of the output light intensity at a column of points (dashed lines) at - $x_{r}$ obtained when performing the binary identity function using digital holographic logic.

the fact that the high spatial frequencies corresponding to the border of the light modulating elements were not passed by the optical computer lens system. An improvement in performance was suggested and demonstrated by Dr. Ronald Grosso of Perkin-Elmer with the results shown in Figure 13. In this case the 1 's complement of the binary identity function, namely, the Exclusive OR, was created. This was done by translating $\mathrm{H}_{\mathrm{abr}}$ by one half cycle of the spatial frequency corresponding to the final term in equation (9). This has the effect of producing a relative phase shift of $180^{\circ}$ between the output generated from the input at $x_{a}$ wi $h$ respect to that generated from the input at $x_{b}$. Thus all regions of constructive interference are replaced by regions of destructive interference and vice versa. The only regions of constructive interference exist where the values of th nut variables are unequal in phase. As can be seen the "znal to noise ratio is considerably enhanced. Thus is would appear that an inverting 
his page is reproduced at the back of the report by a different
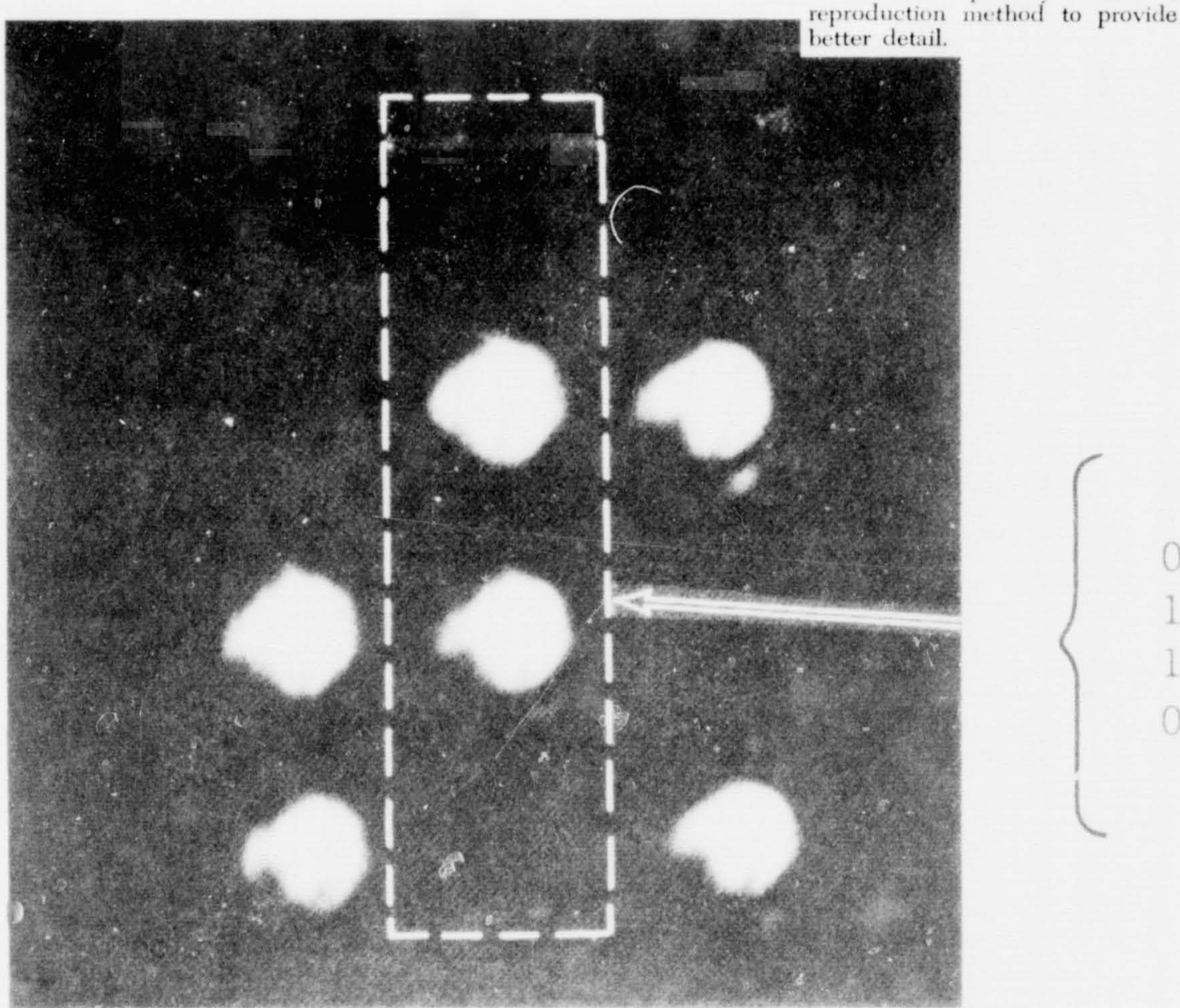

Figure 13. Photomicrograph of the light intensity in the output plane at point - $x$, (dashed lines) obtained when performing the Exclusive OR function using digital holographic logic.

logic corresponding to the I's complement is more practical to use in optical computers performing digital holographic logic than the direct logic whose results are shown in Figure 12.

\section{OPTICAL, TO OPTICAL CONVERSION}

In order to make coherent optical computers using digital holographic logic a practical reality it is necessary to obtain OTTO's for transducing the light intensity distribution in the output plane into appropriate input signals for the next block in the computer chain. One possible OTTO is shown schematically in Figure 14. This device is the PMLM (Photosensitive Membrane Light 


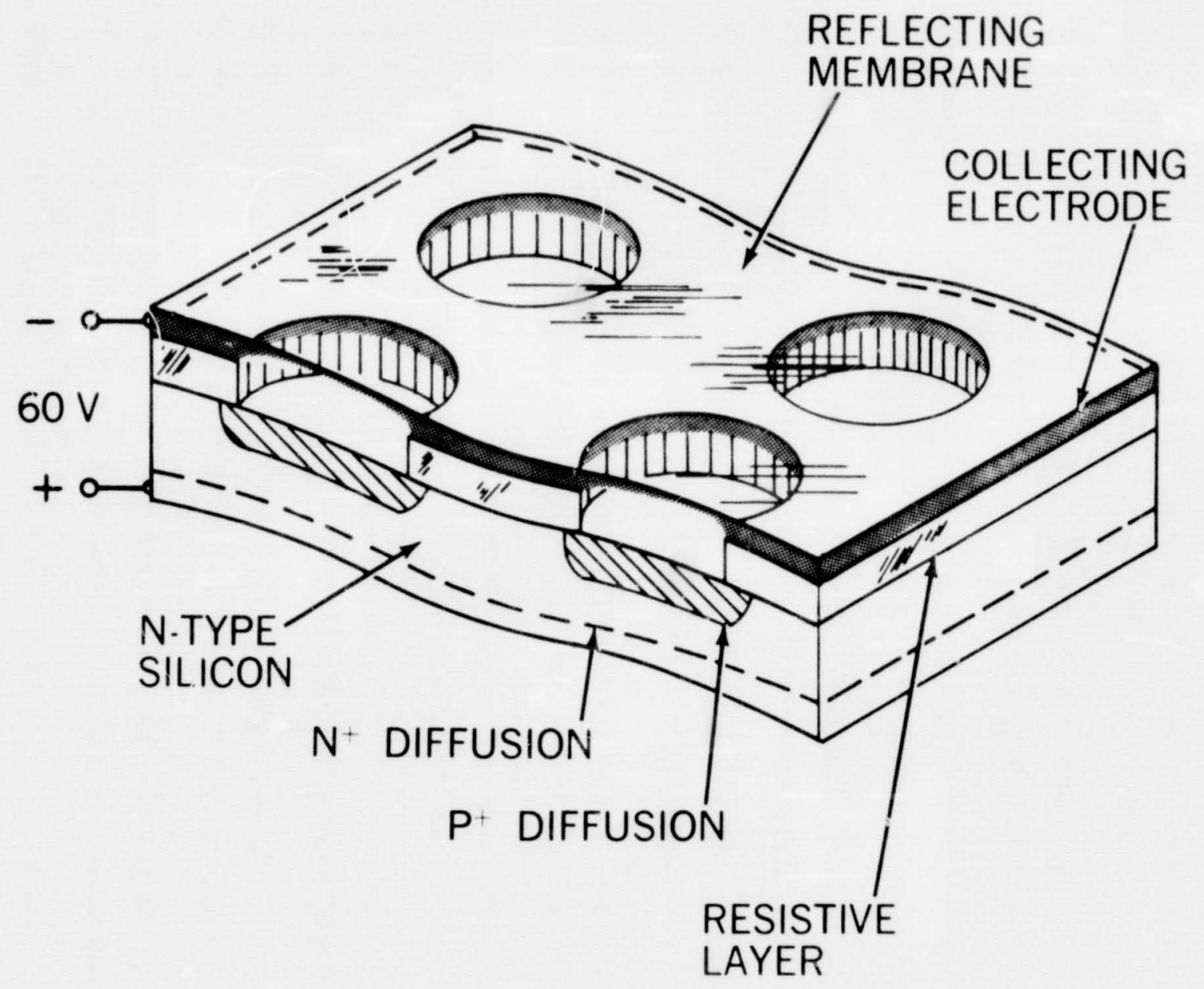

Figure 14. Isometric drawing showing the basic configuration of the photo sensitive membrane light modulator (input surface below; output surface above).

Modulator) originally described by Reizman (5). The PMLM is made from a thin silicon substraie using integrated circuit technology. When n-type silicon is used, a heavy $n+$ diffusion is made on the input surface to act as a transparent electrode. On the opposite surface p+ diffusions are made having the size and spacing of the required input array of phase modulating elements. These diffusions form an array of pn junction diodes. Overlaying the diodes is a resistive layer consisting of a semi-insulating material approximately $1 \mathrm{~m}$ thick. This layer is interrupted with circular perforations registered with the $\mathrm{p}$ regions of the diode array.

Three more layers complete the device. First there is a coilecting electrode on top of the semi-insulating layer (but not extending over the circular 
perforations), next is a polymer membrane which is stretched tightly across the perforations, and finally, a layer of metalization which makes the polymer membrane reflective.

The PMLM is energized by a power supply poled so as to back bias all the diodes in the array. This places the positive terminal of the power supply on the transparent electrode and the negative terminal is common to both the collecting electrode and the membrane metalization. When the input surface of the PMLM is not illuminated, all diodes are back biased and, ideally, no current flows through the semi-insulating layer. This means that the entire potential of the power supply appears across the pn junctions leaving all p regions at the potential of the collecting electrode (and the membrane metalization). When light strikes localiy in the vicinity of one diode, this diode conducts and the $\mathrm{p}$ region now becomes more positive. This introduces a potential difference between the $\mathrm{p}$ region and the membrane metalization. Due to electrostatic forces the membrane locally deflects. Phase modulation of reflected light results from this deflection.

The membrane surface of the PMLM is the output surface and it is coherently illuminated from an optical source. By adjusting the optical computer parameters properly, phase modulation due to deformations of the membrane elements may be made to correspond to the $0^{\circ}$ and $180^{\circ}$ points necessary for doing binary logic holographically. Interferograms showing the operation of two elements of an early PMLM are shown in Figure 15. Each interferogram is of the same portion of the PMLM. Two circular elements are shown which are approximately $40 \mu \mathrm{m}$ in diameter on approximately $100 \mu \mathrm{m}$ centers. From
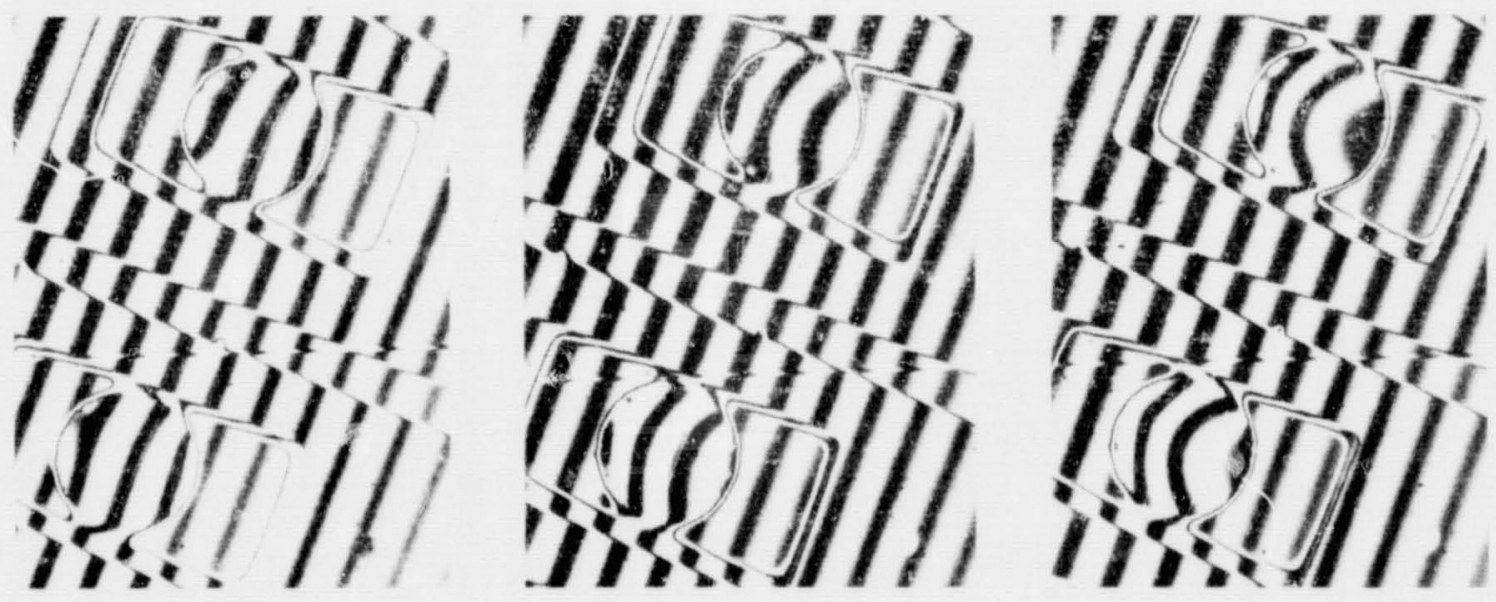

Figure 15. Interferomicrograms showing deflections of the output surface of a photo sensitive membrane light modulator (left to right): power off; power on, no illumination; power on and illuminated. 
left to right in Figure 15 the first interferogram shows the membrane elements undeflected when no power is applied to the PMLM. With the power supply adjusted to 70 volts, both elements deflect due to spurious leakage current through the corresponding diodes. The deflection produces a phase modulation of the order of $180^{\circ}$. This phase level must therefore be utilized as the zero degree phase reference. When the input surface of the PMLM is illuminated, a further deflection takes place as is shown in the right hand interferogram. With proper design a further increase of $180^{\circ}$ may be achieved. Thus the actual operating states of the membrane elements in question would be $180^{\circ}$ to $360^{\circ}$ rather than from $0^{\circ}$ to $180^{\circ}$.

\section{CONCLUSION}

Although all propositional logic may not be carried out using the identity function or the exclusive $O R$, it is possible to expand the above techniques into a complete logical set by incorporating the logical OR function. To accomplish this one must use a three-state logic employing three input elements for each two-input iunction. One element is always at zero degrees of phase. The hologram used is made with three input elements at $0^{\circ}, 120^{\circ}$, and $240^{\circ}$. The input/ output truth table is as follows:

\begin{tabular}{rrl} 
Input A & Input B & Output \\
\hline $0^{\circ}$ & $0^{\circ}$ & Zero \\
$0^{\circ}$ & $180^{\circ}$ & Finite \\
$180^{\circ}$ & $0^{\circ}$ & Finite \\
$180^{\circ}$ & $180^{\circ}$ & Finite
\end{tabular}

The initial entry in the truth table causes the hologram to create a coherent summation in the output plane of three equal vectors of phase $0^{\circ}, 120^{\circ}$, and $240^{\circ}$, respectively. This produces a null condition (destructive interference) and therefore a 0 output. In all other cases, where $180^{\circ}$ is added to either or both of the inputs, the vector summation in the output plane is no longer null and a finite output occurs.

Using this simple modification of the original two-state logic described above, all propositional logic may be performed using the appropriate hologram.

\section{REFERENCES}

1. Preston, K., Jr., Coherent Optical Computers, Ch. 6, McGraw-Hill (1972).

2. Ibid., Ch. 4 . 
3. Vander Lugt, A., "Signal Detection by Complex Spatial Filtering," IEEE Trans. Inform. Theory IT-10, 139 (1964).

4. Leith, E. M., "Reconstructed Wavefronts and Communication Theory," J. Opt. Soc. Amer. 52 (10), 1123 (1962).

5. Reizman, F., "An Optical Spatial Phase Modulator Array Activated by Optical Signals," AGARD Conf. on Opto-El. Sig. Proc. Tech., Oslo, Norway (September 1969).

6. Golay, M. J. E., "Hexagonal Parallel Pattern 'Transformations," IEEE Trans, on Comp. $\mathrm{C}-18,733$ (1969).

7. Preston, K., Jr., "Use of the Golay Logic Processor in Pattern-Recognition Studies Using Hexagonal Neighborhood Logic," Computers and Automata, Polytechnic Press (1972). 